\title{
LÓGICA NEBULOSA PARA AVALIAR RISCOS NA AUDITORIA*
}

FUZZY LOGIC FOR RISK ASSESSMENT IN AUDITING

\author{
JERÔNIMO ANTUNES \\ Professor Doutor do Departamento de Contabilidade e Atuária \\ da Faculdade de Economia, Administração e Contabilidade da \\ Universidade de São Paulo - Campus Capital \\ E-mail: antunes@usp.br
}

\section{RESUMO}

A avaliação dos riscos de que os controles internos de uma entidade possam falhar constitui-se em significativo desafio para os auditores independentes de demonstrações contábeis. As metodologias de trabalho empregadas para tal finalidade, normalmente, utilizam a lógica clássica, ou também denominada binária, presumindo que os fatores de riscos estão presentes, ou não, em um determinado tipo de processo de controle. O objetivo deste trabalho foi conceber um modelo de avaliação de risco dos controles internos de uma entidade utilizando a lógica nebulosa (fuzzy logic), para contemplar os elementos difusos que compõem os fatores desse tipo de risco analisados na auditoria de demonstrações contábeis. A validação conceitual do modelo concebido foi realizada por meio de entrevistas e debates com especialistas em auditoria de demonstrações contábeis e com consultas a bibliografias relevantes pertinentes. Como conclusão do estudo, ficou patente que o modelo de avaliação de risco, com o uso da lógica nebulosa, elimina a restrição binária da lógica clássica e permite tratar, de forma quantitativa, conceitos ambíguos através da aplicação de uma escala psicométrica, para refletir predicados tais como: "muito bom", "bom", "razoável", "de grande importância", "de pouca importância" etc., tendo potencial para produzir resultados mais amplos e próximos da realidade.

Palavras-chave: Avaliação de Risco de Controle; Lógica Nebulosa; Contabilidade; Auditoria Independente de Demonstrações Contábeis.

\section{ABSTRACT}

The assessment of the risks that an entity's internal control system may fail represents a significant challenge to independent auditors. The methodologies used to audit financial statements are usually supported by classical logic, also called binary logic, departing from the relatively simplistic premise that risk factors are either present or not in a certain kind of control process. This study aimed to conceive a risk assessment model for an entity's internal control system, using the fuzzy logic approach, to take into account the diffuse elements that compose the factors of this type or risk, which are analyzed in financial statements auditing. The conceived model was conceptually validated though interviews and debates with financial statement auditing experts and relevant bibliography. We concluded that the use of fuzzy logic to support risk assessment models not only eliminates the binary restriction imposed by classic logic, but also allows for the quantitative treatment of ambiguous concepts through a psychometric scale, to reflect adjectives like "very good", "good", "reasonable", "of great importance", "of little importance" etc. This approach makes it possible to produce broader results that are closer to reality.

Keywords: Control risk assessment; Fuzzy logic; Accounting; Independent audit of financial statements. 


\section{INTRODUÇÃO}

Opinar sobre algo envolve, inerentemente, o risco de acertar ou errar. No caso de auditores independentes, esse risco se traduz na manifestação, através de um parecer, sobre se as demonstrações contábeis de uma entidade representam adequadamente, em todos os aspectos relevantes, a posição patrimonial e financeira dessa, o resultado das suas operações, as mutações do seu patrimônio líquido e as origens e aplicações de seus recursos, de acordo com os princípios de contabilidade geralmente aceitos, ou não.

Para poder expressar uma opinião, os auditores necessitam coligir um conjunto de informações (evidências), que considerem apropriado e suficiente e que consiga mitigar, a um nível aceitável, todas as incertezas que decorrem de um processo de avaliação de fatos e eventos, expressos sob o formato de elementos contábeis, tais como: ativo, passivo, receitas, despesas etc.

O estudo do sistema de controle interno é o ponto de partida lógico para a auditoria de demonstrações contábeis e parece-nos irrecusável admitir que os auditores são inevitavelmente dependentes da qualidade do sistema de controle interno da entidade auditada, assim como é perceptível o grau de correlação entre a gestão do risco das transações levadas a efeito no mundo empresarial (isto é, o risco assumido pelo tomador de decisões na empresa) e a qualidade dos controles que o previne, corrige e revela.

O American Institute of Certified Public Accountants - AICPA registra a importância desse assunto na segunda norma de trabalho de campo conforme tradução livre de Antunes (1 998:45):

Um entendimento suficiente da estrutura de con-

trole interno deve ser obtido para planejar a auditoria

e determinar a natureza, oportunidade e extensão dos

testes por serem realizados.

Como se pode depreender, o resultado da avaliação dos riscos dos sistemas de controles internos de uma entidade é componente significativo no processo de determinação da natureza, época e extensão dos testes de auditoria por serem aplicados pelo auditor independente de demonstrações contábeis.

O modelo de avaliação de risco de auditoria', de acordo com a revisão bibliográfica e as pesquisas de campo realizadas no bojo deste trabalho, é parte fundamental da metodologia de trabalho dos auditores independentes $e$ utiliza como ferramenta, em essência, uma matriz de risco emanada da expressão matemática

$$
\mathrm{AR}=\mathrm{IR} \times \mathrm{CR} \times \mathrm{AP} \times \mathrm{TD},
$$

em que: $A R=$ Audit Risk; $I R=$ Inherent Risk; $C R=$ Control Risk; $\mathrm{AP}=$ Analytical Procedures e $\mathrm{TD}=$ Test of Details, apresentada no apêndice da norma de auditoria Statements on Auditing Standards - SAS 39 - Audit Sampling (AU 350.48) emitida pelo American Institute of Certified Public Accountants - AICPA e usam como sustentáculo a lógica clássica, em que os elementos que compõem os fatores de riscos são avaliados de forma binária, qual seja, estão presentes ou não em um determinado tipo de controle, existem ou não, são realizados ou não, e assim por diante. Todavia, esse julgamento apóia-se em diversos elementos ambíguos, que não têm as características de verdades pontuais e definitivas e que, portanto, precisam ser interpretados de maneira mais ampla que não aquela restrita permitida pela lógica clássica.

Por outro lado, um método quantitativo identificado na revisão bibliográfica deste trabalho que freqüentemente é utilizado em estudos e aplicações práticas sobre processos de decisão em situações de incerteza ou de ambigüidade e capaz de trabalhar com uma grande quantidade de conceitos difusos, tais como: muito alto, alto, mediano, baixo, fraco, razoável e similares, com frequêencia usados no processo de avaliação de risco de controle interno em auditoria independente de demonstrações contábeis, é a lógica nebulosa (fuzzy logic).

O desafio que se apresenta, então, é a construção de um modelo conceitual que permita ao auditor independente avaliar, com o auxílio da lógica nebulosa, de maneira sistematizada e racional os riscos dos sistemas de controles internos de uma entidade, no âmbito dos exames de auditoria de demonstrações contábeis, contemplando os aspectos ambíguos e incertos inerentes ao ambiente da operação e aos elementos dos processos de controle. Em decorrência, os resultados produzidos poderiam ter alcance mais amplo e maior aproximação à realidade (e, portanto, com mais eficácia no desenvolvimento dos trabalhos do auditor independente) quando realizada com o modelo construído.

Para tanto, faz-se necessário identificar os elementos relevantes, de natureza precisa ou ambígua, normalmente utilizados no processo de aferição de controle interno pelos auditores independentes e presentes nos seus métodos de trabalho, bem como da literatura profissional especializada e, a partir daí, edificar um modelo conceitual estruturado para permitir um processo de avaliação com a utilização dos conceitos e dos mecanismos da lógica nebulosa.

I De acordo com Boynton (2002:29I), "Risco de Auditoria é o risco de que o auditor possa inadvertidamente não modificar adequadamente seu parecer sobre demonstrações contábeis que contêm erros ou classificações indevidas materiais". 


\section{LÓGICA NEBULOSA PARA REDUÇÃO DE INCERTEZAS}

Os conjuntos nebulosos e a lógica nebulosa (originalmente Fuzzy Logic) possibilitam a geração de técnicas eficazes para a solução de problemas de naturezas diversas e autores, tais como: Chandra, Dubois, Korvin, Siegel, Sriram e outros, relatam inúmeras aplicações nas áreas de sistemas especialistas, computação com palavras, raciocínio aproximado, linguagem natural, robótica e, no contexto que nos interessa, nas áreas de controle de processos e de tomada de decisões.

A revisão bibliográfica, também, apontou que a lógica nebulosa foi aplicada de forma pioneira em meados da década de 70, na área de controle de processos industriais, especificamente em uma máquina de vapor, experiência conduzida em Londres, no Queen College, pelo professor Abe Mamdani. A primeira aplicação industrial significativa ocorreu na mesma época na Dinamarca, tendo como usuários a indústria de cimento F.L. Smidth Corporation. Atualmente, diversos produtos bem conhecidos da população mundial operam com tecnologia fundamentada na lógica nebulosa, dentre os quais Shaw (1999) relaciona: câmeras de vídeo, aparelhos de ar condicionado, fornos de microondas, máquinas de lavar roupa, incineradores de lixo e outras aplicações.
A lógica nebulosa torna-se cada vez mais importante como ferramenta capaz de capturar informações vagas, ambíguas ou imprecisas, geralmente descritas em linguagem natural para transformá-las em forma numérica, permitindo ampla aplicação em ambientes informatizados e de Inteligência Artificial. O objetivo dessa lógica é fornecer os fundamentos para efetuar o raciocínio aproximado com proposições imprecisas, usando a teoria dos conjuntos nebulosos como ferramenta principal, conforme explica Shaw (1999).

A proposta da lógica nebulosa é assumir uma premissa que varia em grau de pertinência, no intervalo de 0 a I, o que leva o elemento do conjunto nebuloso a ser parcialmente verdadeiro, ou parcialmente falso.

Nguyen e Walker (2000) afirmam que a lógica nebulosa é um superconjunto da lógica booleana tradicional, por estender o conjunto restrito dessa, composto da dualidade "verdadeiro" ou "falso", representado por I ou 0, acrescentando-lhe o conceito de parcialmente verdadeiro, isto é, valores entre o completamente verdadeiro (de valor I) e o completamente falso (de valor 0 ). De fato, uma das principais características da lógica booleana é o axioma do Terceiro Excluído, qual seja, não há alternativa para um valor verdade além do par "verdadeiro" e "falso".

\section{O CONTROLADOR DE LÓGICA NEBULOSA PARA APLICAÇÕES PRÁTICAS}

A transformação de todos os conceitos, vistos até agora, em um mecanismo de aplicação prática foi o desafio a que se propuseram diversos pesquisadores, dentre eles o Prof. Mamdami, que projetou um modelo, denominado originariamente Fuzzy Logic Controller, e que inspirou muitos trabalhos e vem comumente demonstrado, com ou sem adaptações, em diversas obras literárias a respeito do tema. A Figura I, a seguir, apresenta um modelo conceitual de como opera o Controlador de Lógica Nebulosa.

Note-se que o mecanismo primordial desse modelo consiste em "fuzzificar", ou seja, introduzir ao universo nebuloso, as variáveis discretas, representadas por escalas numéricas, processá-las com base em regras estabelecidas com o auxílio de informações de especialistas e, em segui-

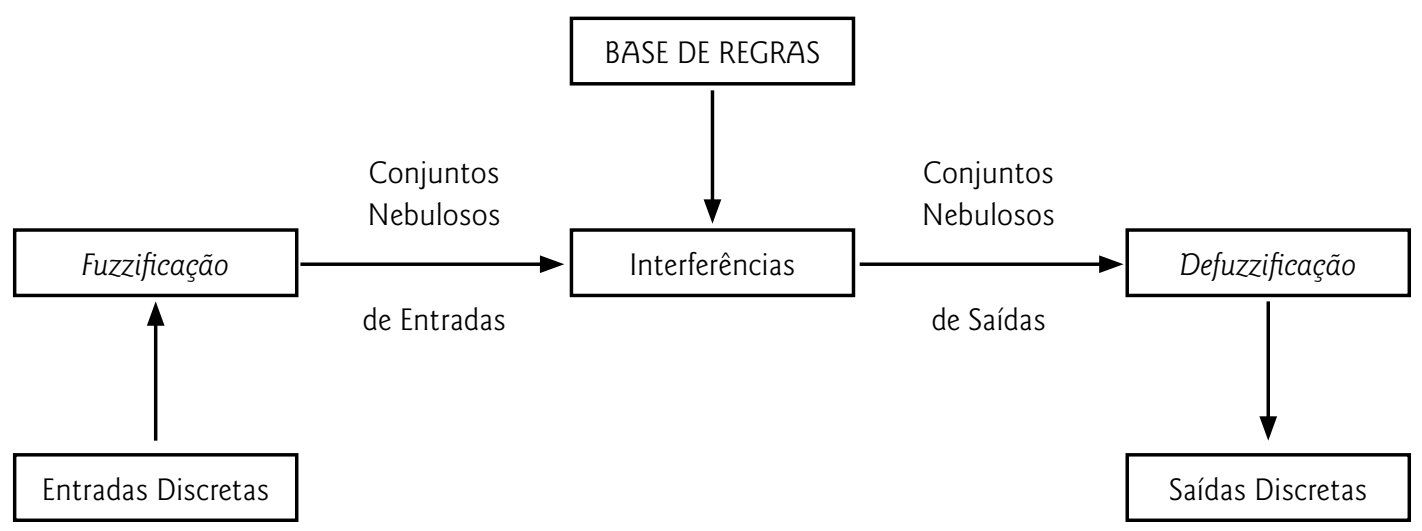

Fonte: Adaptada de Bojadziev (1997).

Figura 1

Estrutura de um Controlador de Lógica Nebulosa 
da, "defuzzificar", o que significa resgatá-las no formato de saídas discretas, ou seja, em números representativos para um processo de tomada de decisão.

As Entradas Discretas são as variáveis lingüísticas, atreladas a algum tipo de escala numérica (por exemplo, uma temperatura de $25^{\circ} \mathrm{C}$ ).

A "Fuzzificação" promoverá a transformação de entradas discretas em entradas nebulosas (fuzzy inputs), considerando, por exemplo, os intervalos de graus Celsius que rotulam as temperaturas ambientais em fria, fresca, normal, morna e quente. Assim, por exemplo, se for imputado ao sistema a informação de que a temperatura ambiente é de $33^{\circ} \mathrm{C}$, o processo de "fuzzificação" indicará que essa é "quente" com um grau de pertinência de 0,46 e "morna" com um grau de 0,20.

Uma vez obtidas as entradas nebulosas, o modelo deverá realizar as inferências necessárias para gerar as saídas dos conjuntos nebulosos. Esse processo de inferência consiste na aplicação das Regras de Controle, também conhecidas como Regras de Produção, sobre as entradas nebulosas e sua conseqüente avaliação e informação dos resultados inferidos, ainda sob a forma de conjuntos nebulosos.

Nesse momento, normalmente, são consultados especialistas na matéria sob a qual o controlador da lógica nebulosa vai operar, para definição das regras. Essas são de natureza condicional ou incondicional. Para as regras de natureza condicional, o formato utilizado para expressá-las utiliza a sintaxe: Se (premissa), Então (conclusão). Por exemplo: Se a temperatura é alta Então acione o sistema de ventilação.
Para as regras de natureza incondicional, não é utilizada a segunda parte condicionante, ou seja, o termo Então. Assim, a expressão simplesmente realiza uma asserção. No exemplo dado, a regra incondicional seria, assim, expressa: A temperatura é alta.

As regras podem conter mais de uma condicionante. Tomando como referência o tema deste trabalho, poderse-ia exemplificar essa condição como segue: SE a Atribuição de Autoridade e Responsabilidade é ruim E a Importância para os Controles é grande, ENTÃO o Risco da Infra-Estrutura é alto.

As premissas da regra são denominadas Antecedentes e a ação estabelecida é chamada de Conseqüente. As premissas são relacionadas pelos conectivos lógicos, os operadores nebulosos conhecidos como Operador de Conjunção (E) ou Operador de Disjunção $(\mathrm{OU})$. O primeiro está associado à operação de Intersecção dos conjuntos nebulosos e o segundo à operação de União desses conjuntos.

Os procedimentos de inferência consistem na avaliação das variáveis antecedentes pelas Regras de Produção estabelecidas. Para tanto, as seguintes etapas devem ser realizadas pelo Controlador de Lógica Nebulosa:

- identificar os valores correspondentes aos graus de pertinência dos termos lingüísticos correspondentes às antecedentes;

- determinar a força das conclusões de cada regra disparada;

- definir a saída nebulosa.

\section{MODELO DE AVALIAÇÃO DE RISCO DE CONTROLE USANDO A LÓGICA NEBULOSA}

A construção do modelo conceitual de avaliação de riscos de controle para uso em auditoria independente de demonstrações contábeis tomou como referência primária a estrutura básica estabelecida no relatório Internal Control - Integrated Framework do Committee of Sponsoring Organizations of Treadway Commission - COSO e adotada pelo American Institute of Certified Public Accountants - AICPA, na sua norma SAS-78 - Consideration of Internal Control in a Financial Statement Audit: An Amendment to SAS n ${ }^{\circ} 55$ e na norma ISA-400 - Risk Assessments and Internal Control da International Federation of Accountants ${ }^{2}$.

Faz-se relevante ressaltar que o desenvolvimento do modelo conceitual, em tela, foi ajustado com base no estudo e avaliação dos manuais internos descritivos das estratégias, dos métodos e procedimentos adotados nos trabaIhos de avaliações de riscos de auditoria de demonstrações contábeis das principais empresas de auditoria que atuam no Brasil (dentre elas, três das quatro maiores do mundo, denominadas "big four") visitadas em nossa pesquisa de campo e que franquearam o acesso, e, ainda, com base nas compilações dos resultados das indagações a respeito do modus operandi nesse assunto, dessas e de outras que não permitiram o acesso direto aos seus compêndios.

Uma vez concluído, o esboço do modelo conceitual foi submetido à apreciação e análise de alguns profissionais das empresas de auditoria pesquisadas, que, como experts nos processos de avaliação de riscos de controle, contribuíram com diversas sugestões de aperfeiçoamento.

\section{1 Construção da Estrutura Conceitual Básica do Modelo}

A estrutura do modelo conceitual considera que um sistema de controle interno de uma entidade tem cinco componentes, a saber: Ambiente de Controle, Avaliação de Riscos, Atividade de Controle, Informação e Comunicação e Monitoração. De acordo com a norma SAS-78 do AICPA, cada componente envolve várias políticas e procedimentos de controle, necessários para que sejam atingidos os objetivos primordiais do sistema de controle interno de uma entidade, que se configuram como a necessidade de proporcionar segurança razoável na confiabilidade das informações financeiras por tal sistema gerado, na obediências às leis e regulamentos

2 Cabe enfatizar que a metodologia de trabalho da maioria das empresas visitadas segue, em termos gerais, a estrutura acima referida, porém com maior aderência quanto à avaliação de risco inerente e de ambiente de controle. 
aplicáveis à entidade e na geração de condições para ela operar com eficácia e eficiência. Os componentes de controles internos são, assim, entendidos pelo COSO:

- Ambiente de Controle: Compreende e reflete as atitudes dos gestores e suas crenças e valores quanto à importância do sistema de controle interno para a entidade, influenciando e embasando todos os demais componentes que nele operam, bem como a consciência das pessoas envolvidas.

- Avaliação de Risco: São as atividades desenvolvidas pela entidade para a identificação, análise e administração dos riscos que possam causar impacto relevante nos negócios e nos controles internos e na elaboração das demonstrações contábeis em conformidade com os princípios de contabilidade geralmente aceitos.

- Atividades de Controle: Compreendem o conjunto de políticas e procedimentos que proporcionam condições para assegurar que as diretrizes emanadas da administração estejam sendo aplicadas e obedecidas.

- Informação e Comunicação: São os métodos estabelecidos para identificar, reunir, analisar, classificar e relatar as transações da entidade tempestivamente e em formato tal que impeçam erros ou classificações indevidas nas asserções da administração contidas nas demonstrações contábeis e que possibilitem, ainda, que as pessoas cumpram suas responsabilidades com os controles internos.

- Monitoração: São os processos que avaliam a qualidade do desempenho dos controles internos ao longo do tempo, contemplando análises sobre o desenho dos sistemas, a tempestividade de operação dos controles e a adoção de ações corretivas.
Para compatibilizar o modelo conceitual de avaliação de risco de controle com os fundamentos da lógica nebulosa e, principalmente, com o modelo Controlador de Lógica Nebulosa e suas regras de acionamento, foram utilizados os seguintes recursos:

- Aglutinação dos componentes do controle interno em duas grandes categorias: Risco do Ambiente de Controle e Risco dos Outros Componentes do Controle.

- Segregação dos Riscos do Ambiente de Controle e do Risco dos Outros Componentes do Controle em sete Classes de Riscos, que foram assim denominadas: Gestão de Pessoas, Modelo de Decisão, Infra-Estrutura, Avaliação de Risco, Atividades de Controle, Informação e Comunicação e Monitoramento.

Para as Classes de Riscos do Risco dos Outros Componentes de Controle foram tomadas como referência a segregação proposta pelo AICPA para os componentes de controle interno. Assim, tomando como exemplo a Classe de Risco de Gestão de Pessoas, os Fatores de Riscos identificados para ela compreendem a avaliação dos efeitos da postura das pessoas na organização, decorrentes da integridade moral e da conduta ética, do compromisso em realizar suas tarefas de forma competente, das políticas de recrutamento, seleção e de orientações sobre os papéis e responsabilidades do funcionário na organização e outras necessárias para a eficaz gestão de pessoas. O Quadro I 0 apresenta os Fatores de Riscos e

\begin{tabular}{|l|l|}
\hline \multirow{4}{*}{ Glasses de Riscos } & Fatores de Riscos \\
\hline \multirow{4}{*}{ Modelo de Decisão } & Integridade e Valores Éticos \\
\cline { 2 - 2 } & Comprometimento com Competência \\
\cline { 2 - 2 } & Políticas e Práticas de Recursos Humanos \\
\hline \multirow{2}{*}{ Infra-Estrutura } & Filosofia e Estilo Operacional da Administração \\
\cline { 2 - 2 } & Postura para Informações Contábeis \\
\cline { 2 - 2 } & Conselho de Administração e Comitê de Auditoria \\
\hline \multirow{2}{*}{ Avaliação de Risco } & Atribuição de Autoridade e Responsabilidade \\
\cline { 2 - 2 } & Estrutura Organizacional \\
\hline \multirow{3}{*}{ Atividades de Controle } & Ameaças Internas \\
\cline { 2 - 2 } & Ameaças Externas \\
\hline \multirow{2}{*}{ Informação e Comunicação } & Restrições de Acessos e Funções \\
\cline { 2 - 2 } & Controles de Processamento de Informações \\
\cline { 2 - 2 } & Revisões de Desempenho \\
\cline { 2 - 2 } & Processamento da Informação \\
\hline \multirow{2}{*}{ Monitoramento } & Divulgação da Informação \\
\cline { 2 - 2 } & Monitoramento Interno \\
\cline { 2 - 2 } & Supervisão Externa \\
\hline
\end{tabular}

Fonte: Elaborado pelo autor.

Quadro 1 Classes de Riscos e Fatores de Riscos de Controle 
as respectivas Classes de Riscos:

Os Fatores de Riscos considerados foram classificados com denominações criadas a partir de discussões com auditores entrevistados e que se alinham ao conteúdo dos Elementos de Avaliações, exemplificados pelo AICPA na norma citada. Os Elementos de Avaliação foram compilados a partir dos exemplos fornecidos pelo AICPA e pelo COSO, bem como do conteúdo dos questionários de avaliações ou dos manuais de auditoria das empresas visitadas. Para viabilizar a operacionalização do modelo conceitual, os citados elementos de avaliação foram condensados ou desdobrados, de forma muitas vezes diferente das fontes, porém seguindo uma seqüência lógica que permita não perder a finalidade e utilidade do quesito.

O Quadro 20 exemplifica alguns Fatores de Risco e os seus Elementos de Avaliação.
O modelo concebido utiliza três espécies de rótulos, sendo dois deles compostos de cinco termos (rótulos) e um composto de três termos. São os seguintes:

- Muito Bom, Bom, Razoável, Ruim e Péssimo Esses rótulos são aplicados aos Elementos de Avaliação. Assim, por exemplo, o auditor designará que o elemento "Segregação de Funções", em uma escala numérica previamente estabelecida de 0 a 100 (poderia ser qualquer outra escala), mereceria o valor 75 .

- Grande, Média e Pequena Estabelecem a importância atribuída pela crença do avaliador aos Fatores de Riscos. Assim, por exemplo, o avaliador pode julgar, considerando todas as evidências de que dispõe, que a importância, por exemplo, da atuação do Conselho de Administra-

\begin{tabular}{|c|c|}
\hline Fatores de Risco & Elementos de Avaliação \\
\hline \multirow{3}{*}{ Integridade e Valores Éticos } & Comunicação dos valores e do código de ética da entidade. \\
\hline & Fornecimento de orientação moral para todos colaboradores. \\
\hline & Eliminação de incentivos para atos ilegais, antiéticos ou desonestos. \\
\hline \multirow{3}{*}{ Comprometimento com Competência } & Conhecimentos e habilidades necessários para a competência exigida. \\
\hline & Treinamento dos funcionários para desenvolvimento das funções. \\
\hline & Experiência necessária para a execução das tarefas. \\
\hline \multirow{3}{*}{ Políticas e Práticas de Recursos Humanos } & Políticas de recrutamento e seleção adequadas para contratar funcionários. \\
\hline & Orientação sobre a cultura da organização, papéis e responsabilidades. \\
\hline & Processo de avaliação de desempenho para promoções e remunerações. \\
\hline \multirow{3}{*}{ Filosofia e Estilo Operacional da Administração } & Forma de assunção e monitoramento dos riscos dos negócios. \\
\hline & Forma de tratamento dispensado aos principais executivos da entidade. \\
\hline & Atitudes para com as pessoas das áreas de informática e contabilidade. \\
\hline \multirow{3}{*}{ Postura para Informações Contábeis } & Escolha conservadora ou agressiva dos princípios contábeis. \\
\hline & Processo de determinação de estimativas contábeis. \\
\hline & Atitudes e ações para o processo de elaboração de demonstrações contábeis. \\
\hline \multirow{3}{*}{ Conselho de Administração e Comitê de Auditoria } & Independência em relação à diretoria, experiência e estatura empresarial. \\
\hline & Envolvimento com atividades estratégicas e adequação de ações. \\
\hline & Natureza e extensão da integração com os auditores internos e independentes. \\
\hline \multirow{3}{*}{ Atribuição de Autoridade e Responsabilidade } & Definição da maneira como, e a quem, atribuir autoridade e responsabilidade. \\
\hline & Atribuição de responsabilidades aos gestores pela consecução de resultados. \\
\hline & Responsabilidade por documentação, transações e acessos - ambiente de TI. \\
\hline \multirow{3}{*}{ Estrutura Organizacional } & Estruturas hierárquicas apropriadas ao tamanho da entidade. \\
\hline & Organograma formal pertinente com a natureza da atividade. \\
\hline & Estrutura organizacional eficaz na geração de informação contábil. \\
\hline \multirow{3}{*}{ Ameaças Internas } & Capacidade de identificar riscos das alterações no ambiente operacional. \\
\hline & Capacidade de identificar riscos de novas tecnologias e novos funcionários. \\
\hline & Detecção de efeitos provocados por mudanças nos sistemas de informações. \\
\hline \multirow{3}{*}{ Ameaças Externas } & Capacidade de identificar ameaças e oportunidades por rápido crescimento. \\
\hline & Capacidade de identificar riscos por novas atividades, linhas ou produtos. \\
\hline & Reconhecimento oportuno dos efeitos de novos pronunciamentos contábeis. \\
\hline
\end{tabular}




\begin{tabular}{|c|c|}
\hline Fatores de Risco & Elementos de Avaliação \\
\hline \multirow{3}{*}{ Restrição de Acessos e Funções } & Segregação de funções entre execução, registro e custódia de ativos. \\
\hline & Controle de acesso físico aos ativos tangíveis. \\
\hline & Acesso aos controles e informações estratégicas e operacionais. \\
\hline \multirow{3}{*}{ Controles de Processamento de Informações } & Delegação de autorizações das transações e controles pertinentes. \\
\hline & Controle das aprovações de alterações de sistemas de informações. \\
\hline & Integridade e exatidão das informações fornecidas pelos controles internos. \\
\hline \multirow{3}{*}{ Revisões de Desempenho } & Elaboração de orçamentos e nível de análises dos gestores. \\
\hline & Correlações de informações operacionais, econômicas e financeiras. \\
\hline & Comparações das contagens físicas com registros contábeis e fiscais. \\
\hline \multirow{3}{*}{ Processamento da Informação } & Processamento das transações válidas, integrais, tempestivas e corretas. \\
\hline & Fornecimentos de trilhas de auditoria completas pelo sistema contábil. \\
\hline & Documentos hábeis para comprovar transações e arquivos adequados. \\
\hline \multirow{3}{*}{ Divulgação da Informação } & Plano de contas contábeis apropriado para as atividades da entidade. \\
\hline & Manual de políticas e principais práticas contábeis adotadas. \\
\hline & Relatórios contábeis e financeiros completos, oportunos e objetivos. \\
\hline \multirow{2}{*}{ Monitoramento Interno } & Avaliação do desenho dos sistemas de controles internos. \\
\hline & Estrutura organizacional, atuação e resultados da Auditoria Interna. \\
\hline \multirow{2}{*}{ Supervisão Externa } & Implantação de melhorias de controles sugeridos pela Auditoria Externa. \\
\hline & Aperfeiçoamentos de controles internos requeridos por agências reguladoras \\
\hline
\end{tabular}

Fonte: Elaborado pelo autor.

Quadro 2 Alguns Fatores de Risco e seus Elementos de Avaliação

ção e do Comitê de Auditoria, em uma escala de 0 a 100, também previamente estabelecida, situa-se no valor 80

As Figuras 2, 3 e $4 \bullet$ demonstram, graficamente, a concepção do modelo de avaliação de risco de controle. A Figura 2 refere-se ao processo de avaliação do Risco do Ambiente de Controle, enquanto a Figura 3 demonstra a avaliação do Risco dos Outros Componentes do Controle.

A Figura 4 mostra o encerramento de todo o processo, avaliando o Risco de Controle, em razão dos dois principais tipos de riscos das duas figuras anteriores, calculado pela fórmula:

$$
\mathrm{RC}=\frac{(\mathrm{RAC} * 3)+(\mathrm{ROC} * 2)}{5},
$$

em que: $\mathrm{RC}=$ Risco de Controle; $\mathrm{RAC}=$ Risco do Ambiente de Controle e ROC $=$ Risco de Outros Componentes do Controle.

Os pesos atribuídos aos fatores são decorrentes de julgamento de especialistas consultados quanto à importância que o Risco do Ambiente de Controle tem em rela- ção ao Risco de Outros Componentes do Controle.

Além de utilizar a escala de valores e atribuir o grau de pertinência aos termos lingüísticos, o modelo necessita das Regras de Produção para combinar as diversas possibilidades de rótulos resultantes das inferências deduzidas dos Elementos de Avaliação, dos Fatores de Riscos, das Classes de Riscos e dos Tipos de Riscos.

Dessa forma, a inferência de que o Elemento de Avaliação "Comunicar Valores e Código de Ética" é rotulado com o adjetivo "Bom", combinado com o termo lingüístico "Muito Bom" do elemento "Fornecer Orientação Moral" e com o rótulo "Razoável" aplicado para o elemento "Eliminar Incentivos e Tentações", resulta no qualificativo "Bom" para o Fator de Risco "Integridade e Valores Éticos". Como se depreende, as regras utilizadas são do tipo "Se"...."Então".

O quadro 3 mostra algumas das 125 combinações possíveis nesse exemplo.

A definição dessas regras é, normalmente, realizada com a ajuda de especialistas. Nessa fase de modelagem conceitual, as regras foram discutidas com alguns profissionais de significativa experiência na atividade de auditoria independente, para aquilatar e refinar as expressões antecedentes e conseqüentes das Regras de Produção. 

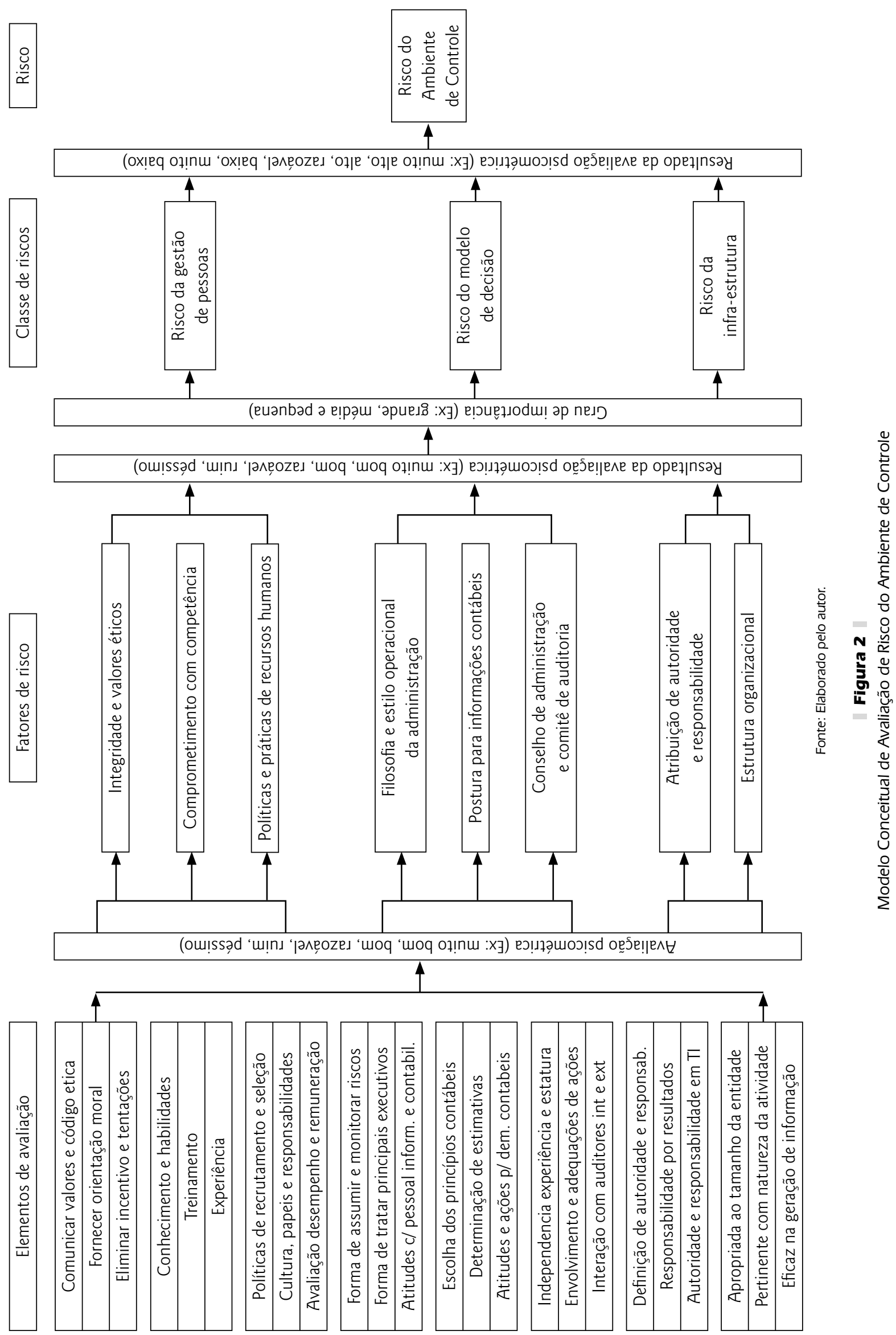

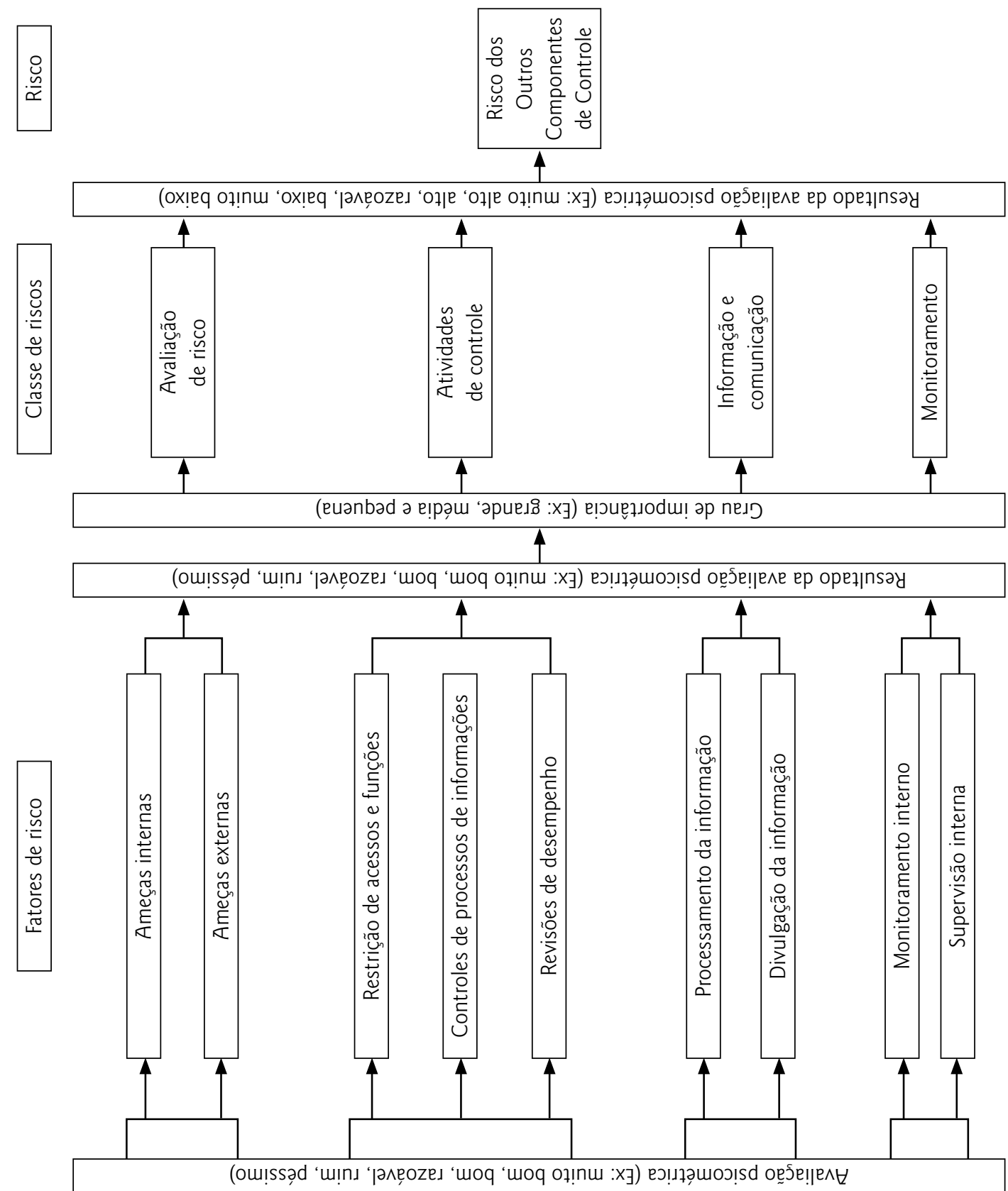

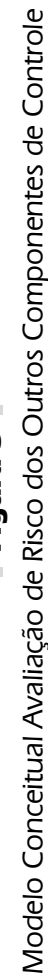

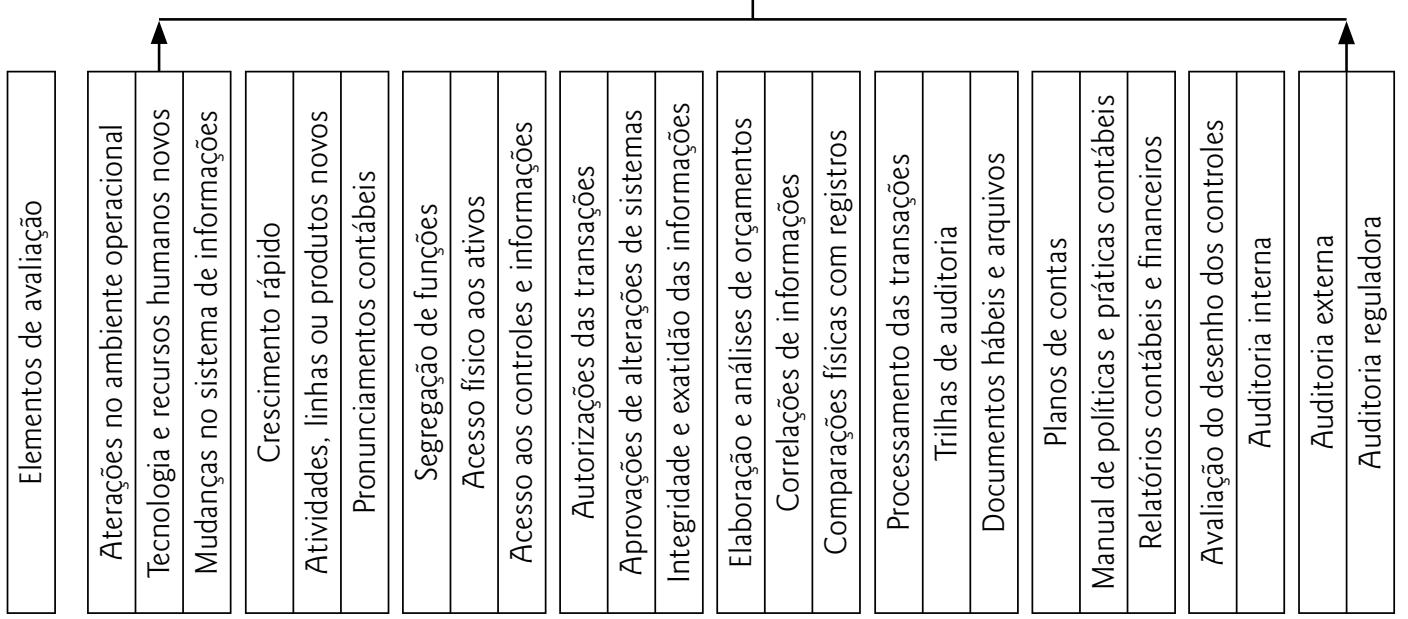



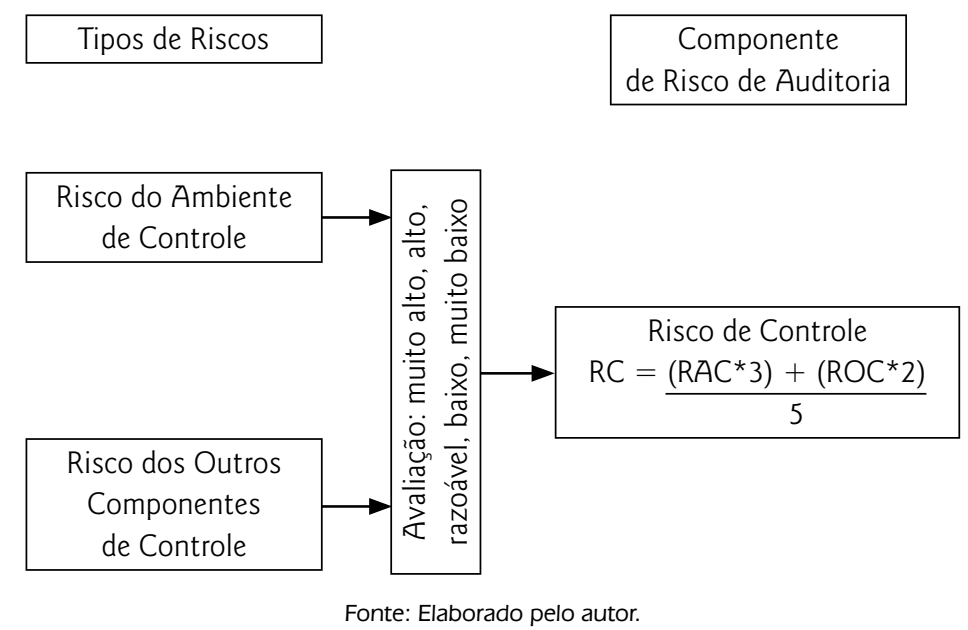

Figura 4

Modelo Conceitual de Avaliação Final de Risco de Controle

\begin{tabular}{|c|c|c|c|}
\hline Elementos de Avaliação & Termo Avaliado & Fator de Risco & Termo Resultante \\
\hline Conhecimento e Habilidade & Ruim & \multirow{3}{*}{$\begin{array}{l}\text { Comprometimento } \\
\text { com Competência }\end{array}$} & \multirow{3}{*}{ Bom } \\
\hline Treinamento & Bom & & \\
\hline Experiência & Muito Bom & & \\
\hline Definição de Autoridade e Responsabilidade & Razoável & \multirow{3}{*}{$\begin{array}{c}\text { Atribuição de Autoridade } \\
\text { e Responsabilidade }\end{array}$} & \multirow{3}{*}{ Razoável } \\
\hline Responsabilidade por Resultados & Péssimo & & \\
\hline Autoridade e Responsabilidade em TI & Bom & & \\
\hline Avaliação do Desenho dos Controles & Muito Bom & \multirow{2}{*}{ Monitoramento Interno } & \multirow{2}{*}{ Razoável } \\
\hline Auditoria Interna & Péssimo & & \\
\hline
\end{tabular}

Fonte: Elaborado pelo autor.

Quadro 3 Exemplos de Combinações de Termos Lingüísticos para Avaliação dos Fatores de Riscos

\section{CONCLUSÕES E SUGESTÕES}

A proposta deste estudo foi a construção de um modelo para avaliação dos riscos de controles internos, no âmbito de um processo de auditoria de demonstrações contábeis, calcado no método quantitativo conhecido como lógica nebulosa e visando possibilitar a contemplação de elementos de avaliação incertos e difusos que compõem, de maneira representativa, os fatores de riscos analisados pelos auditores independentes.

A validação conceitual foi realizada através de debates e consultas a especialistas, obras literárias e alguns manuais práticos de metodologia de trabalho de auditoria. Os tópicos conclusivos decorrentes deste estudo, bem como suas vantagens, são os seguintes:
- o modelo concebido com o uso da lógica nebulosa contempla os aspectos ambíguos e incertos inerentes ao ambiente da operação e aos elementos dos processos de controle, possibilitando a avaliação sistematizada e racional dos riscos dos sistemas de controles internos de uma entidade para o escopo da auditoria independente de demonstrações contábeis;

- o modelo elimina a restrição binária de respostas "sim" ou "não" para questionários de avaliação de risco que, preponderantemente, sustentam as metodologias de trabalho das empresas de auditoria independente pesquisadas, possibilitando incor- 
porar respostas com maior amplitude, tais como: "freqüentemente sim", "nem sempre" etc, que são traduzidas para uma escala numérica previamente fornecida, em que o "sim" estaria no início da contagem (zero, por exemplo) e o "não" estaria ao final da escala ( 100 , por exemplo), deixando o meio para o apontamento das respostas intermediárias entre o positivo e o negativo, conforme melhor refletir o julgamento do avaliador;

- a lógica nebulosa permitiu tratar, de forma numérica, predicados tais como: "bom”, "razoável”, "de grande importância", por meio da aplicação de uma escala psicométrica;

- como decorrência desses adequados tratamentos, o resultado da avaliação é expresso em um valor numérico que representa o risco de controle avaliado em uma entidade, no curso de uma auditoria de demonstrações contábeis e pode ser utilizado como o fator RC na função de avaliação do risco de auditoria $\mathrm{RA}=\mathrm{RI}(\mathrm{x}) \mathrm{RC}(\mathrm{x}) \mathrm{RD}$, orientada pelo AICPA e base dos processos largamente empregados pelos auditores independentes nesse tipo de auditoria.

Um aspecto que pode ser observado como uma limitação do modelo desenvolvido é que sua aplicação não elimina o elemento "julgamento profissional" que está na base de todo o processo de avaliação de risco de controle, em qualquer metodologia de trabalho empregada pelos auditores independentes.

O potencial de aplicação de versões ampliadas e aperfeiçoadas do modelo em questão, assim como da lógica nebulosa e de outros métodos quantitativos em processos de auditoria independente que podem ser vislumbrados seriam, como exemplo, os seguintes:

- a estrutura conceitual pode ser ampliada para incorporar novos elementos de avaliação de riscos e até mesmo conceitos, como temporariamente ocorre com edições de pronunciamentos de entidades reguladoras, tais como o COSO, IFAC, AICPA, IBRACON etc.;
- o modelo pode agregar elementos mais detalhadose específicos, voltados para a avaliação das atividades de controle $e^{3}$, normalmente agrupadas nos métodos tradicionais de trabalho das empresas de auditoria em ciclos operacionais, como, por exemplo, o ciclo de Compras e Desembolsos, de Receitas, de Recursos Humanos etc. Poder-se-ia, ainda, vincular essas avaliações detalhadas com as asserções contidas nas contas e classes de transações das demonstrações contábeis, tais como: propriedade, existência, ocorrência etc.;

- os elementos de avaliação e o resultado de um modelo com a mesma tipologia podem estar voltados especificamente para a sinalização de ocorrência de fraudes nas demonstrações contábeis ou, até mesmo, para desvios que podem não causar impacto direto, imediato e significativo na posição econômica e financeira da entidade, mas podem gerar prejuízos de imagem, credibilidade e outros com potenciais pecuniários futuros;

- outros métodos quantitativos, tais como as redes neurais e os algoritmos genéticos, poderiam ser aplicados em modelos de avaliações de risco de controle, tendo como base o presente modelo, ou desenvolvidos para aplicações similares em processos de auditagem.

Finalizando, espera-se que o presente estudo possa contribuir para a Ciência Contábil e mais especificamente para o campo da auditoria das demonstrações contábeis, à medida que visou demonstrar a viabilidade da aplicação prática de um instrumento das Ciências Exatas para tentar mensurar elementos típicos das Ciências Sociais Aplicadas. Naturalmente, em razão do seu caráter inovador, tanto a proposta do estudo em si, como o modelo conseqüente gerado, demandam o envolvimento de pesquisadores também de outras disciplinas para melhorar e ampliar as oportunidades de aplicação na atividade de auditoria e outras similares.

\section{Referências Bibliográficas}

AMERICAN INSTITUTE OF CERTIFIED PUBLIC ACCOUNTANTS - AICPA. AICPA Professional Standards - Vol. 1 - U.S. Auditing Standards - Attestation Standards. New York: AICPA, 1997.

ANTUNES, Jerônimo. Contribuição ao Estudo da Avaliação de Risco e Controles Internos na Auditoria de Demonstrações Contábeis no Brasil. São Paulo, 1998. Dissertação (Mestrado em Contabilidade e Controladoria) - Departamento de Contabilidade e Atuária da Faculdade de Economia, Administração e Contabilidade da Universidade de São Paulo, São Paulo.

BOJADZIEV, George; BOJADZIEV, Maria. Fuzzy Logic for Business, Finance, and Management. Singapure: World Scientific, 1997.

CHANDRA, Akhilesh; AGRAWAL, Surrenda P.. A Fuzzy Decision Support for Cost Management Systems Design. In: SIEGEL, Philip H. KORVIN, Andre de, OMER, Khursheed. Applications of Fuzzy Sets and the Theory of Evidence to Accounting, II. Stanford: JAI Press, 1998. p. $99-115$.

3 Um dos cinco componentes do controle, segundo o COSO e o AICPA e que no modelo desenvolvido é considerado uma das Classes de Risco que compõem o Risco dos Outros Componentes de Controle. 
COMMITTEE OF SPONSORING ORGANIZATIONS OF THE TREADWAY COMMISSION - COSO. Internal Control: Integrated Framework. USA, 1994.

DUBOIS, Didier; PRADE, Henri. Fuzzy Sets and Systems: Theory and Applications. Volume 144 in Mathematics in Science and Engineering. San Diego: Academic Press, Inc., 1980.

INSTITUTO BRASILEIRO DE CONTADORES - IBRACON. Normas Internacionais de Auditoria e Código de Ética Profissional. São Paulo, 1998.

KORVIN, Andre de; et al.. Modeling Job Scheduling for Audit Uncertainties Using Fuzzy Logic. In: SIEGEL, Philip H. KORVIN, Andre de, OMER, Khursheed. Applications of Fuzzy Sets and the Theory of Evidence to Accounting, II. Stanford: JAI Press, 1998. p. 39-54.

NGUYEN, Hung T.; WALKER, Elbert A.. A First Course in Fuzzy Logic. 2nd. Edition. Boca Raton: Chapman \& Hall/CRC, 2000.

SHAW, Ian S.. Controle e Modelagem Fuzzy. São Paulo: Edgard Blucher, 1999.

SIEGEL, Philip H.; RIGSBY, John T.; BOURGEOIS, Brian. An Application of Fuzzy Set Theory to the Problem of Evaluating Net Realizable Value of Accounts Receivable. In: SIEGEL, Philip H. KORVIN, Andre de, OMER, Khursheed. Applications of Fuzzy Sets and the Theory of Evidence to Accounting, II. Stanford: JAI Press, 1998. p. 89-102.

SRIRAM, Ram S.; WHEELER, Patrick. Uncertainty Handling in Accounting Systems: A Comparison of Alternative Approaches to Knowledge Representation. In: SIEGEL, Philip H. et al.. Applications of Fuzzy Sets and the Theory of Evidence to Accounting. Stanford: JAI Press, 1995. p.163-186.

\section{NOTA - Endereço do autor}

Universidade de São Paulo

Faculdade de Economia, Administração e Contabilidade

Departamento de Contabilidade e Atuária
Av. Prof. Luciano Gualberto, 908 - prédio 3

Cidade Universitária

São Paulo - SP

05.508-900 